\title{
44616 - CE-BASED DETERMINATION OF WHOLE-BLOOD PROPOFOL CONCENTRATION
}

\author{
Koen Raedschelders, Anesthesiology, Pharmacology, and Therapeutics. The University of \\ British Columbia, Vancouver, BC, Canada; \\ David Ansley, Dep't of APT The University of British Columbia; \\ David Chen, Dep't of Chemistry The University of British Columbia; \\ Hong Zhang, Chemistry The University of British Columbia; \\ Hao Yang, Chemistry The University of British Columbia; \\ $N$ Fang, Chemistry The University of British Columbia;
}

\begin{abstract}
INTRODUCTION: Our research group is interested in potential pleurotropic pharmacological activities associated with anesthetic agents. Early cell culture and isolated heart model studies from our lab suggested that propofol might be cardioprotective during cardiopulmonary bypass, provided an adequate concentration is achieved. In order to further investigate the dosedependent in vivo effects of this drug, we developed a novel Capillary Electrophoresis-based method capable of quantitatively measuring propofol concentrations in whole blood.

METHODS: Local IRB approval was obtained for this study. The preparative step involves the addition of a known amount of thymol to the sample as an internal standard, followed by cell lysis, and subsequent liquid extraction. The sample is then dried under nitrogen flow and resuspended in $10 \%$ acetonitrile.

The separative phase uses a Capillary Electrophoresis mode called Micellar Electrokinetic Capillary Chromatography. The separating ability of this technique has two components: 1) the discriminative migration of the analytes in an electric field of $500 \mathrm{~V} / \mathrm{cm}$, and 2) the extent to which the analytes interact with micelles during the separation. Quantification involves relating the peak area ratio to a standard curve.
\end{abstract}

RESULTS: Although the sample preparation step is somewhat time consuming, many samples can be processed simultaneously. The actual separation is complete in less than 25 minutes. Our results indicate that propofol can safely be stored at -80 degrees celcius and subsequently processed simultaneously. Our method requires 500 microL of whole blood. Organic solvent and buffer volumes are equally scaled down. Our limit of detection is in the range of $150 \mathrm{ng} / \mathrm{ml}$. This method can produce sharp baseline-resolved peaks for both thymol and propofol in less than 25 minutes and is linear at clinically relevant propofol concentrations.

DISCUSSION: This method is capable of quantitatively determining the clinically relevant concentration of propofol in whole blood. The pharmacokinetics of propofol dictate that infusion algorithms do not necessarily correlate with whole blood target achieved concentrations. This discrepancy limits our ability to interpret a larger spectrum of potentially cardioprotective effects. As such, our method is suitable for in vivo investigations into possible concentrationdependent pleurotropic effects of this drug. Similarly, the speed and efficiency of this type of separation, combined with recent advances in microfluidic separation devices, makes this method a steppingstone towards improved target controlled infusion devices. 